\title{
Nonlinear Seismic Response Analysis of the Surrounding Rock-Tunnel System in the Mountain Areas Under SV Wave
}

\author{
Dongrui XIA ${ }^{\text {a,1 }}$, Lijiang HAN ${ }^{\text {a }}$, Yang LI $^{\text {a }}$, Lichuang MA ${ }^{\text {a }}$ and Junjie YAN ${ }^{\text {a }}$ \\ ${ }^{\mathrm{a}}$ The Third Construction Engineering Company LTD. of China Construction Second \\ Engineering Bureau, 10070 Beijing, China
}

\begin{abstract}
The seismic responses and failure mechanisms of the tunnels embedded in the rock are quite different from those of the aboveground structures due to the dynamic interactions between tunnel and surrounding rock. In the previous studies, the tunnel models were under some extent of simplification without considering much of critical issues such as the three-dimensional (3D) characteristics, nonlinear mechanical properties or initial in-situ stress in the model, which are bound to bring the unpredictable errors in the evaluation of seismic response of tunnel-rock system. In this paper, some 3D nonlinear finite element models are established to evaluate the seismic response of surrounding rock-tunnel system in the mountain areas, considering the initial stress state of surrounding rock-tunnel system induced by gravity and excavation, General Mohr Coulomb nonlinear constitutive. Based on the proposed model, the optimal value of the longitudinal length of the model is firstly discussed to determine the value range of the model size. After that, a series of numerical parametric analyses are carried out to investigate the deformation of the surrounding rock. One important finding is that there exists a most unfavorable stress condition which makes the tunnel induce maximum seismic responses. Finally, the typical control variable method is employed to compare the results of the models established in this paper with those of the model considering or not some of significance factors, the comparison results further prove the necessity of establishing the 3D nonlinear model.
\end{abstract}

Keywords. Seismic response, mountain tunnel, parameter analysis, most unfavorable position

\section{Introduction}

Earthquake will seriously threaten the safety of human life and property once it happens. Therefore, the seismic response and failure mechanism of structures under the action of earthquake need to be conducted urgently [1]. However, the seismic response and the failure mechanism of underground structures are quite different from those of the aboveground structures due to the existence of the surrounding rock and soil. As a consequence, the seismic response and the failure mechanism of the underground structures under seismic action need to be investigated systematically.

${ }^{1}$ Dongrui Xia, The Third Construction Engineering Company LTD. of China Construction Second Engineering Bureau, 10070 Beijing, China; E-mail: xiadongrr@126.com. 
At present, the model test method is restricted to the problems of implementation cost and scale ratio and the analytical method is unable to analyze the complicated problems such as nonlinear. The numerical simulation method can be used to analyze the problem of complex field and nonlinear problems, which plays an important role in the aseismic research. Sica et al. [2] used finite element method to assess the effects of space and depth of the cavities on the ground motion amplification. Narayan et al. [3] developed a P-SV wave finite difference algorithm with lined and unlined tunnels. Kim SH [4] studied the 3-D underground structures with inelastic material behavior under dynamic loading. Alielahi et al. [5] investigated the seismic response of a linear elastic medium subjected to vertically incident SV and $\mathrm{P}$ waves by BEM.

In this paper, some 3D finite element models of surrounding rock-tunnel system are firstly established, considering the initial in-situ stress balance and the process of tunnel excavation. Subsequently the numerical parametric analysis is carried out by ABAQUS software to study the deformation of surrounding rock. According to the calculation results, the most unfavorable position of the tunnel under the action of earthquake is determined. Finally, the control variable method is used to compare the results of the model established in this paper with those of the model not considering some of significance factors.

\section{Numerical Model and Parameters}

\subsection{Establishment of FE Model and Materials Properties}

In the model, the surrounding rock is firstly simulated by the linear elastic constitutive model to roughly determine the value range of longitudinal direction of the model. Then, the Mohr Coulomb (MC) nonlinear constitutive model is selected to consider the nonlinear response of surrounding rock in the subsequent parametric analysis.

According to the MC criterion, the shear strength of soil is expressed as follows:

$$
\tau_{f}=\sigma \cdot \tan \varphi+c
$$

where, $\tau_{f}$ represents shear strength of soil; $\sigma$ is the normal stress acting on the shear plane; $\varphi$ is internal friction angle of soil; $c$ is cohesion of surrounding rock. The mathematical expression of the MC yield failure criterion can be derived by equation 2:

$$
\frac{\sigma_{1}-\sigma_{3}}{2}=\frac{\sigma_{1}+\sigma_{3}}{2} \sin \varphi+c \cos \varphi
$$

In the dynamic analysis of underground structure, Rayleigh damping is often adopted to take into account the energy dissipation of surrounding rock. According to this damping model, damping matrix $\mathbf{C}$, mass matrix $\mathbf{M}$, and stiffness matrix $\mathbf{K}$ satisfy the relation as presented in equation 3 :

$$
\mathbf{C}=\alpha_{R} \mathbf{M}+\beta_{R} \mathbf{K}
$$

with 


$$
\alpha_{R}=\frac{2 \xi}{\omega_{1}+\omega_{2}}, \beta_{R}=2 \xi \frac{\omega_{1}}{\omega_{1}+\omega_{2}}, \xi_{\mathrm{i}}=\frac{\alpha_{R}}{2 \omega_{\mathrm{i}}}+\frac{\beta_{R} \omega_{\mathrm{i}}}{2}
$$

where $\alpha_{R}$ is the proportionality coefficient related to mass in Rayleigh damping; $\beta_{R}$ is the proportionality coefficient related to stiffness in Rayleigh damping; $\xi_{i}$ is critical damping ratio of surrounding rock; $\omega_{i}$ is natural vibration frequency of undamped surrounding rock model. Let $\xi_{1}=\xi_{2}=0.02$ during calculation.

The tunnel structure is simulated by $\mathrm{C} 40$ concrete, whose constitutive model is selected as linear elastic constitutive model. The detailed parameters of surrounding rock and $\mathrm{C} 40$ concrete are shown in table 1 . The inner diameter and the lining thickness of the tunnel is $5 \mathrm{~m}$ and $0.35 \mathrm{~m}$, respectively. The model as shown in figure 1 . Both of the tunnel lining and the surrounding rock are simulated by solid 8 -node element. The mesh size of the element is required to be approximately $1 / 10-1 / 8$ of the seismic wavelength.

Table 1. Physical and mechanical parameters of the surrounding rock and lining.

\begin{tabular}{|c|c|c|c|c|c|}
\hline Materials & $\begin{array}{l}\text { Elastic Modulus } \\
(\mathrm{GPa})\end{array}$ & Poisson's ratio & $\begin{array}{l}\text { Density } \\
\left(\mathrm{kN} / \mathrm{m}^{3}\right)\end{array}$ & $\begin{array}{l}\text { Cohesion } \\
(\mathrm{MPa})\end{array}$ & $\begin{array}{l}\text { Internal } \\
\text { friction angle } \\
\left({ }^{\circ}\right)\end{array}$ \\
\hline Surrounding rock & 2.8 & 0.3 & 20 & 0.8 & 35 \\
\hline Lining & 33 & 0.2 & 25 & & \\
\hline
\end{tabular}

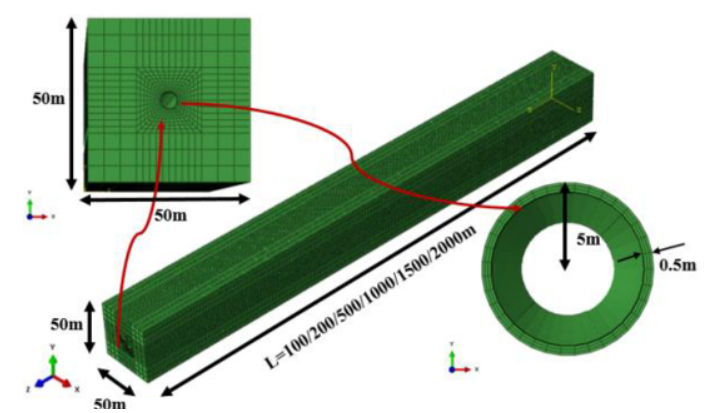

Figure 1. The finite element model of surrounding rock-tunnel system.

\subsection{Boundary Conditions and Earthquake Motion Input}

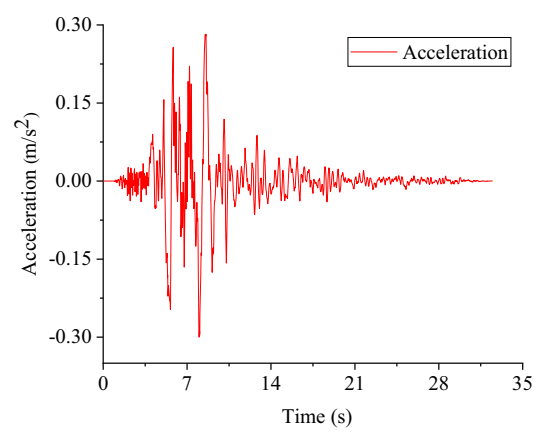

Figure 2. Acceleration time history of Kobe wave. 
In the process of seismic analysis, it is more complicated to employ artificial boundaries to the 3D model especially the complex geological conditions. The normal fixed constraint method is adopted in this paper [6] as a result of time-saving and being implemented directly by commercial software without any programming operation.

The measured seismic wave of Kobe University is selected as the input of ground motion in this paper. The amplitude of the acceleration time history is adjusted to $0.3 \mathrm{~g}$ and the first $32.5 \mathrm{~s}$ is used for calculation. The acceleration time history of Kobe University seismic wave is shown in figure 2.

\subsection{Analysis Procedure}

The main analysis procedures of this paper are as follows:

(1) Establishing the geometric models, dividing the grid and imposing the boundary conditions in the ABAQUS software;

(2) Conducting the initial stress balance in the model;

(3) Excavating stepwise the tunnel under the static general step;

(4) Calculating the equivalent input seismic loads and enforcing it on the bottom of the model by normal fixed constraint method;

(5) Solving the model by the conventional implicit or explicit time integration algorithm.

\section{Results and Discussion}

\subsection{The Selection of the Calculation Length}

In this section, the finite element models with 6 different longitudinal values including $100 \mathrm{~m} ; 200 \mathrm{~m} ; 500 \mathrm{~m} ; 1000 \mathrm{~m} ; 1500 \mathrm{~m}$ and $2000 \mathrm{~m}$ are established, respectively. The optimal tunnel length is selected by the time history response of the vault of the tunnel in the middle cross section.

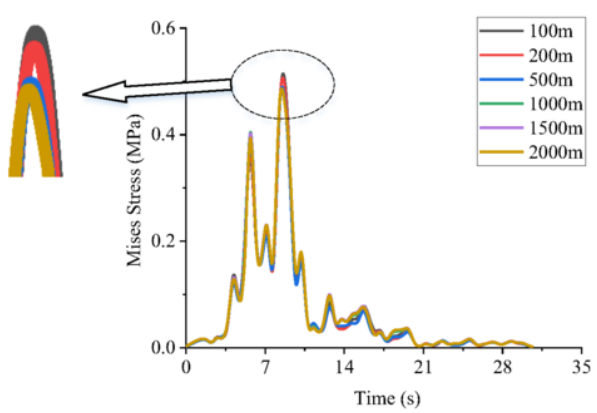

Figure 3. Mises stress time history curve.

It can be seen from figure 3 that the stress time-history curves of tunnels almost coincide with the tunnel length increasing from $1000 \mathrm{~m}$ to $2000 \mathrm{~m}$. With the increase of the length, the seismic response in the middle section of the tunnel is gradually stable duo to the dissipation effect of surrounding rock on the ground motion. As a 
consequence, the tunnel length is set as $1000 \mathrm{~m}$ for the purpose of time-saving and accuracy in the following study.

\subsection{The Response of the Tunnel after Excavation}

It is essential to carry out a balance of the initial stress state of tunnel to obtain the accurate response of tunnel under earthquake motion. The plastic zone distribution and vertical displacement of surrounding rock after tunnel excavation are calculated and analyzed in this section, As shown in figure 4(a), the plastic zones within a certain range of the surrounding rock are produced after excavation, and the largest plastic zone happen at the tunnel waist arch, which gradually decrease to 0 from the waist arch to the vault and the bottom arch. As seen from figure 4(b), a certain range of downward and upward displacement occurs in the vault of the tunnel and the bottom of the arch after excavation, respectively, which are within a reasonable range. The reason is that the initial stress balance state of the tunnel is disturbed after excavation and the force direction is towards the excavated area, leading to the upward displacement in the position of the arch bottom.

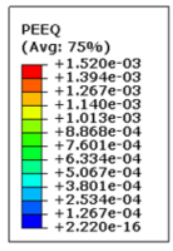

(a)

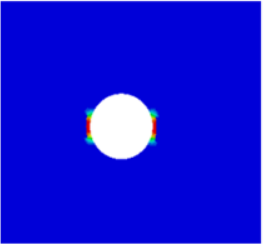

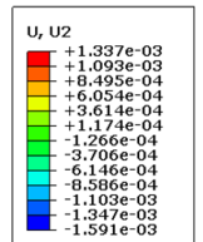

$-1.591 \mathrm{e}-03$

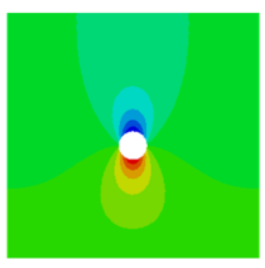

(b)

Figure 4. The response after tunnel excavation: (a) plastic zone and (b) vertical displacement.

\subsection{Longitudinal Seismic Response of Tunnel}

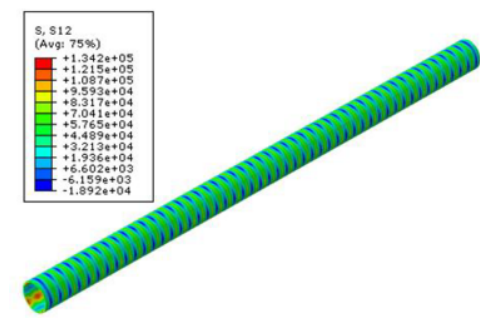

(a)

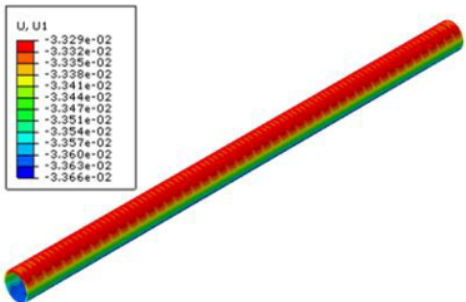

(b)

Figure 5. Longitudinal seismic response of tunnel: (a) shear stress of tunnel, (b) displacement of tunnel.

The tunnel, as a 3D slender structure, is more vulnerable to shear dislocation and axial tensioning failure in the longitudinal direction under earthquake action. Therefore, the shear stress and horizontal displacement of the tunnel are extracted and analyzed in this section. As shown in figures 5(a), the shear stress of the tunnel presents periodic distribution under the action of ground motion, which is the main cause of transverse shear failure. Figures 5(b) describe the horizontal displacement distribution of the tunnel. It can be seen that the displacement of the tunnel shows reciprocating movement. 


\subsection{Transverse Seismic Response of Tunnel}

The failure mechanism of the tunnel is also caused by the transverse failure including includes cracking, ellipsometry, etc. Therefore, the stress on the transverse section of the tunnel is analyzed in this section, as shown in figure 6.

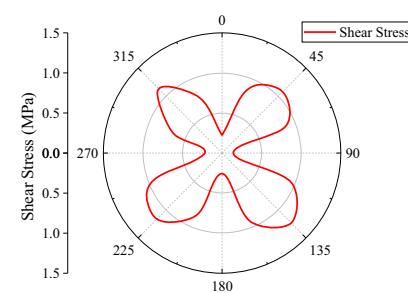

(a)

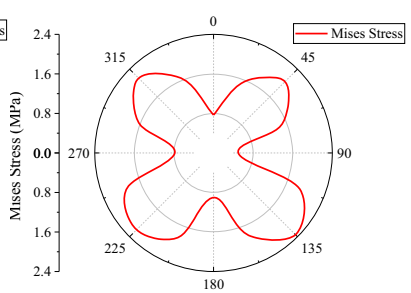

(b)

Figure 6. Transverse seismic response of tunnel: (a) shear stress distribution of tunnel cross section, and (b) Mises stress distribution of tunnel cross section.

Figure 6 (a) shows the shear stress distribution of the cross-section of the tunnel, the maximum stress is larger at $45^{\circ}$ above and $45^{\circ}$ below the left and right sides of the tunnel, and the peak stress occurs at the right arch foot of the tunnel. Figure 6 (b) shows the Mises stress distribution around the tunnel. It can be seen that the Mises stress distribution is similar to the shear stress distribution, and the Mises stress distribution is larger at $45^{\circ}$ above and $45^{\circ}$ below the left and right sides of the tunnel, and the peak stress occurred at the right arch foot of the tunnel. The force is smaller at the top, bottom and both sides of the tunnel. Therefore, the most unfavorable position should be considered in the future seismic design of tunnel cross-section.

\subsection{The Error Analysis}

In this section, the control variable method is adopted to compare the results of the model in this paper (Case 1) with those of not considering the initial stress state (Case 2 ), the non-linearity of soil masses (Case 3) and the 2D model (Case 4). The various cases are shown in table 2 , and the results are shown in figure 7.

Table 2. Four cases in this section.

\begin{tabular}{llll}
\hline & Three-dimension model & Material nonlinear & Initial stress state \\
\hline Case1 & $\sqrt{ }$ & $\sqrt{ }$ & $\sqrt{ }$ \\
Case2 & $\sqrt{ }$ & $\sqrt{ }$ & $\sqrt{ }$ \\
Case3 & $\sqrt{ }$ & $\sqrt{ }$ & $\sqrt{ }$ \\
Case4 & & $\sqrt{ }$ & \\
\hline
\end{tabular}

Figure 7 (a) shows the results of considering excavation and without. The results show that the shear stresses without a consideration of excavation are slightly smaller than those with a consideration of excavation, and the maximum error occurs at the right side of the arch foot. Figure 7 (b) shows the results of considering material nonlinearity and linearity. The results show that considering linearity is slightly smaller than that considering material nonlinearity, and the maximum error occurs at the right side of the arch foot too. Figure 7 (c) shows the results of the 3D model and the 2D 
model. One can find that the results of the 3D model are slightly smaller than those of the 2D model, and the maximum error occurs at the left side of the arch foot.

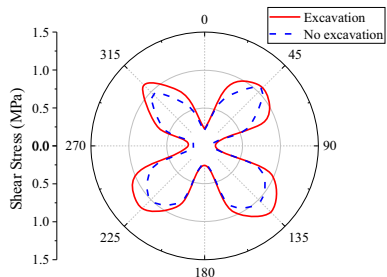

(a)

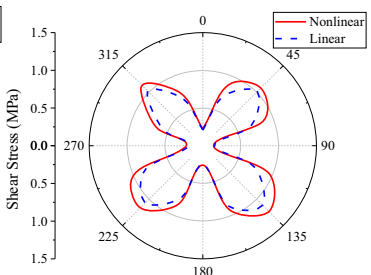

(b)

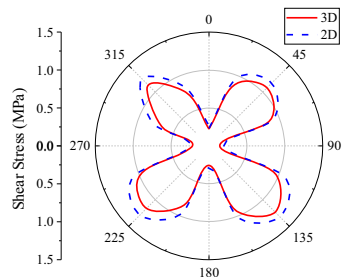

(c)

Figure 7. Comparison of different cases: (a) Excavation and without excavation, (b) nonlinear and linear and (c) 3D model and 2D model

\section{Conclusions}

In this paper, some of significance and relevant conclusions are as follows :(1) the most optimal longitudinal value of the tunnel should be $1000 \mathrm{~m}$; (2) efficiently evaluating the initial stress state of the surrounding rock-tunnel system plays a significant role in obtaining the seismic response of the tunnel more accurately; (3) The stress presents a periodic distribution in the longitudinal seismic response analysis of the tunnel, which is caused by axial tension and compression failure. (4) In the analysis of the cross section, the stress at $45^{\circ}$ above and $45^{\circ}$ below the left and right sides of the tunnel is larger, and the maximum stress occurs at the right arch foot of the tunnel. (5) It can keep the validity and high accuracy for the seismic response of tunnels by the $3 \mathrm{D}$ nonlinear model.

The conclusion of this paper might provide some references for the related research or engineering. Nevertheless, the research in this paper still has some limitations, without considering the nonlinear of concrete material or the different incident angle of seismic motion, which will be full considered in the future work.

\section{References}

[1] Dowding CH, Rozan A. Damage to rock tunnels from earthquake shaking. Journal of the Geotechnical Engineering Division. 1978 Aug; 104(2): 175-191.

[2] Sica S, Dello Russo A, Rotili F, Simonelli AL. Ground motion amplification due to shallow cavities in nonlinear soils. Natural Hazards. 2014 Apr; 71(3): 1913-1935.

[3] Narayan JP, Kumar D, Sahar D. Effects of complex interaction of Rayleigh waves with tunnel on the free surface ground motion and the strain across the tunnel-lining. Natural Hazards. 2015 Oct; 79(1): 479-495.

[4] Kim SH, Kim KJ. Two-phase finite element procedures for dynamic analysis of saturated porous media. Engineering Computations. 2000 Nov; 17(6/7):758-774.

[5] Alielahi H, Kamalian M, Adampira M. Seismic ground amplification by unlined tunnels subjected to vertically propagating SV and P waves using BEM. Soil Dynamics and Earthquake Engineering. 2015 Apr; 71:63-79.

[6] Zienkiewicz OC, Bicanic N, Shen FQ. Advances in computational nonlinear mechanics. In : Doltsinis I.S 1st, editors. Earthquake Input Definition and the Trasmitting Boundary Conditions. Vienna: Springer; 1989. p. 109-138. 